\title{
MicroRNA-155 promotes ox-LDL-induced autophagy in human umbilical vein endothelial cells by targeting the PI3K/Akt/mTOR pathway
}

\author{
SHUANGSHUANG YIN*, SHAONAN YANG* ${ }^{*}$ XUDONG PAN, AIJUN MA, \\ JUANJUAN MA, HAOTIAN PEI, YI DONG, SHU LI, WEI LI and XINRAN BI \\ Department of Neurology, The Affiliated Hospital of Qingdao University, Qingdao, Shandong 266003, P.R. China
}

Received October 31, 2017; Accepted June 7, 2018

DOI: $10.3892 / \mathrm{mmr} .2018 .9236$

\begin{abstract}
Endothelial cell autophagy has a protective role in inhibiting inflammation and preventing the development of atherosclerosis, which may be regulated by microRNA (miR)-155. The present study aimed to investigate the mechanisms of autophagy in the development of atherosclerosis. Human umbilical vein endothelial cells model in vitro and using oxidized low-density lipoprotein (ox-LDL) stimulated cells to simulate the atherosclerosis. MiR-155 mimics, miR-155 inhibitors, and a negative control were respectively transfected in human umbilical vein endothelial cells to analyzed alterations in the expression of miR-155. It was demonstrated that overexpression of miR-155 promoted autophagic activity in oxidized low-density lipoprotein-stimulated human umbilical vein endothelial cells, whereas inhibition of the expression of miR-155 reduced autophagic activity. Overexpression of miR-155 revealed that it regulated autophagy via the phosphatidylinositol-3 kinase $(\mathrm{PI} 3 \mathrm{~K}) / \mathrm{RAC}-\alpha$ serine/threonine-protein kinase (Akt)/mechanistic target of rapamycin pathway (mTOR) signaling pathway. A luciferase reporter assay demonstrated that miR-155 directly bound to the PI3K catalytic subunit a and Ras homolog enriched in brain 3'-untranslated region and inhibited its luciferase activity. Therefore, the results of the present study suggested that miR-155 promoted autophagy in vascular endothelial cells and that this may have occurred via targeting of the PI3K/Akt/mTOR pathway. Thus, miR-155 may
\end{abstract}

Correspondence to: Dr Xudong Pan or Dr Aijun Ma, Department of Neurology, The Affiliated Hospital of Qingdao University, 16 Jiangsu Road, Qingdao, Shandong 266003, P.R. China

E-mail:xdpan022@163.com

E-mail:drmaj@126.com

*Contributed equally

Key words: atherosclerosis, autophagy, microRNA-155, phosphatidylinositol-3 kinase/RAC- $\alpha$ serine/threonine-protein kinase/mechanistic target of rapamycin pathway be considered as a potential therapeutic target for the treatment of atherosclerosis.

\section{Introduction}

Autophagy is a basic cellular maintenance mechanism, by which unnecessary proteins and organelles are degraded in order to maintain cellular survival and homeostasis under various conditions of intracellular stress (1). Autophagy is becoming a focus of research in endothelial cells in regards to atherosclerosis prevention. Endothelial cell injury is a major step in the pathological progression of atherosclerosis. Autophagy may occur to protect the cells following this injury (2). When autophagy fails or is inhibited, the breakdown of endothelium integrity facilitates local lipid deposition, resulting in atherogenesis, plaque instability, acute vascular occlusion and even sudden mortality $(3,4)$. The mechanism of autophagy regulation is complex, and is thought to be linked to multiple signaling pathways (5-7). The phosphatidylinositol-3 kinase (PI3K)/RAC- $\alpha$ serine/threonine-protein kinase (Akt)/mechanistic target of rapamycin (mTOR) signaling pathway is an essential regulator in cellular proliferation, survival, metabolism and autophagy (8). In addition, this pathway may be involved in the induction of autophagy in atherosclerosis $(9,10)$. However, the molecular mechanisms that control endothelial cell autophagy in the development of atherosclerosis remain to be fully elucidated.

MicroRNAs (miRNAs/miRs) are small 19-24 nucleotide non-coding RNAs that function in the post-transcriptional regulation of target gene expression (11). Previous research has revealed that miRNAs are essential for autophagy regulation in atherosclerosis (12-14). Previously, miR-155 has been reported to have a pivotal role in the function of endothelial cell apoptosis regulation, vascular smooth muscle migration, lipid metabolism regulation and the inflammatory response, thus affecting the incidence of atherosclerosis through physiological and pathological processes (15); however, the association between miR-155 and autophagy in endothelial cells has not been fully elucidated. Therefore, the the present study aimed to investigate the role of miR-155 in autophagy.

Our previous study demonstrated that miR-155 promotes oxidized low-density lipoprotein (ox-LDL)-induced autophagy 
in human umbilical vein endothelial cells (HUVECs) (16). The present study investigated whether miR-155 regulated vascular endothelial cell autophagy via the PI3K/Akt/mTOR signaling pathway. Bioinformatic analyses were conducted to predict target genes for miR-155 in the PI3K/Akt/mTOR signaling pathway and it was revealed that PI3K catalytic subunit a (PIK3CA) and Ras homolog enriched in brain (Rheb) were direct targets of miR-155. The results suggested that miR-155 promoted autophagy, and that this may have occurred through the targeting of the PI3K/Akt/mTOR pathway in vascular endothelial cells.

\section{Materials and methods}

Cell culture. HUVECs and 293T cells were purchased from the Shanghai Institute for Biological Sciences, Chinese Academy of Sciences (Shanghai, China). According to the supplier's protocols, cells were cultured in Dulbecco's modified Eagle's medium (Hyclone; GE Healthcare Life Sciences, Logan, UT, USA) supplemented with $10 \%$ fetal bovine serum (Gibco; Thermo Fisher Scientific, Inc., Waltham, MA, USA) and 1\% antimycotic-antibiotic solution (Beijing Solarbio Science \& Technology Co., Ltd., Beijing, China) in a humidified atmosphere of $5 \% \mathrm{CO}_{2}$ at $37^{\circ} \mathrm{C}$.

Cell treatment. miR-155 mimics, inhibitor and negative controls (NCs) were purchased from Guangzhou RiboBio Co., Ltd. (Guangzhou, China). Cells were transfected with $50 \mathrm{nmol} / \mathrm{l}$ miR-155 mimics, miR-155 inhibitor or NCs for 6 h, using Lipofectamine ${ }^{\circledR} 2000$ (Invitrogen; Thermo Fisher Scientific, Inc.) according to the manufacturer's protocol; complete medium containing FBS was then added for $24 \mathrm{~h}$. In order to induce autophagy, HUVECs were stimulated with $100 \mu \mathrm{g} / \mathrm{ml}$ ox-LDL (Guangzhou Yiyuan Biotech Co., Ltd., Guangzhou, China) for $12 \mathrm{~h}$. They were subsequently treated with $0.5 \mu \mathrm{mol} / 1$ bafilomycin $\mathrm{A} 1$ for $12 \mathrm{~h}$ at $37^{\circ} \mathrm{C}$ to block cytolysosome and lysosome fusion. HUVECs were divided into seven experimental groups: i) Control; ii) ox-LDL + miR-155 inhibitor; iii) ox-LDL + miR-155 mimic; iv) ox-LDL + bafilomycin A1; v) ox-LDL; vi) ox-LDL + miR-155 inhibitor-NC; and vii) ox-LDL + miR-155 mimic-NC.

Transmission electron microscopy (TEM). HUVECs were plated in $10 \mathrm{~cm}$ petri dishes in a humidified atmosphere of $5 \% \mathrm{CO}_{2}$ at $37^{\circ} \mathrm{C}$ for $24 \mathrm{~h}$. Cells at $70-80 \%$ confluence were used for experiments. The cells were trypsinized, washed once with PBS and harvested by centrifugation at 20,000 x g at $4^{\circ} \mathrm{C}$ for $10 \mathrm{~min}$. The cell pellet $(0.5 \mathrm{~cm})$ was fixed in $10 \mathrm{ml}$ ice-cold $2.5 \%$ glutaraldehyde in $0.1 \mathrm{M}$ sodium cacodylate buffer ( $\mathrm{pH} 7.2)$ at $4^{\circ} \mathrm{C}$ overnight. Samples were sent to the laboratory of Qingdao University (Qingdao, China), and subsequently post-fixed in PBS with $1 \%$ osmium tetroxide for $1 \mathrm{~h}$ at $4^{\circ} \mathrm{C}$, followed by dehydration in graded ethanol $(70 \%$ for $20 \mathrm{~min}, 96 \%$ for $20 \mathrm{~min}$, and $100 \%$ ethanol for $20 \mathrm{~min}$ twice) and propylene oxide. Cells were embedded in epoxy resin. Sectioned grids were stained with $2 \%$ uranyl acetate in $50 \%$ methanol for $10 \mathrm{~min}$ and lead citrate for $7 \mathrm{~min}$ at $4^{\circ} \mathrm{C}$. Images were captured on a transmission electron microscope (JEM-1220; JEOL, Ltd., Tokyo, Japan) at an accelerating voltage of $80 \mathrm{kV}$ and a magnification of $\mathrm{x} 40,000$. A total of
10 field of view were randomly selected to observe autophagosomes in each group.

Confocal microscopy. Cells $\left(3 \times 10^{4}\right.$ cells per well) were cultured on glass coverslips in a 24-well culture plate covered with cell culture medium (DMEM with $10 \%$ FBS and $1 \%$ antimycotic-antibiotic solution) in a humidified atmosphere of $5 \% \mathrm{CO}_{2}$ at $37^{\circ} \mathrm{C}$. Next, the cells on coverslips were fixed using $4 \%$ paraformaldehyde (Beijing Solarbio Science \& Technology Co., Ltd.) for $15 \mathrm{~min}$ at $4^{\circ} \mathrm{C}$ and permeabilized with $1 \%$ Triton X-100 (Beijing Solarbio Science \& Technology Co., Ltd.) for 10 min. Following washing of the coverslips, cells were blocked with $3 \%$ bovine serum albumin (BSA; Beyotime Institute of Biotechnology, Haimen, China) to prevent non-specific antibody binding and incubated at $4^{\circ} \mathrm{C}$ overnight with $1 \% \mathrm{BSA}$ in PBS $0.5 \%$ Tween containing antibodies against microtubule-associated protein light chain 3 (LC3; cat. no. 3868S; 1:100; Cell Signaling Technology, Inc., Danvers, MA, USA). Cells were subsequently washed twice with PBS $0.5 \%$ Tween and incubated with fluorescein isothiocyanate-conjugated goat anti-rabbit IgG secondary antibodies (1:200; BIOSS, Beijing, China) in $1 \%$ BSA in the dark for $1 \mathrm{~h}$ at $37^{\circ} \mathrm{C}$. The cells were washed in PBS and nuclei were stained with $10 \mu \mathrm{g} / \mathrm{ml}$ of DAPI (Shanghai Yeasen Biotechnology Co., Ltd., Shanghai, China) for $5 \mathrm{~min}$ in the dark. Cells were washed again and antifade mounting medium (Beyotime institute of Biotechnology) was added prior to mounting on glass slides for examination. Images were acquired using a laser-scanning confocal imaging system (magnification, x25,000; TCS SP5; Leica Microsystems GmbH, Wetzlar, Germany).

Western blot analysis. Protein extracts were prepared from cells using radioimmunoprecipitation assay lysis buffer containing protease and phosphatase inhibitors (Beyotime Institute of Biotechnology). Following centrifugation at $4^{\circ} \mathrm{C}$ for $5 \mathrm{~min}$ at $30,000 \mathrm{x} \mathrm{g}$ the supernatant was collected and protein concentrations in the lysates were measured using a bicinchoninic acid protein assay kit (Beyotime Institute of Biotechnology). Total protein (50 mg) was separated by $10 \%$ SDS-PAGE and transferred onto polyvinylidene difluoride membranes, which were blocked with $5 \%$ skimmed milk powder in Tris-buffered saline with $0.1 \%$ Tween 20 (Beijing Solarbio Science \& Technology Co., Ltd.) for $2 \mathrm{~h}$ at $4^{\circ} \mathrm{C}$. The membranes were incubated with the following primary antibodies at $4^{\circ} \mathrm{C}$ overnight: Rabbit anti-LC3B (cat. no. ab48394; Abcam), rabbit anti-PI3K (cat. no. 4257; Cell Signaling Technology, Inc.), rabbit anti-phosphorylated (p)-PI3K (cat. no. 5538; Cell Signaling Technology, Inc.), rabbit anti-Rheb (cat. no. 13879; Cell Signaling Technology, Inc.), rabbit anti-Akt (cat. no. 4691S; Cell Signaling Technology, Inc.), rabbit anti-p-Akt (cat. no. 4060; Cell Signaling Technology, Inc.), rabbit anti-70 kDa ribosomal protein S6 kinase 1 (p70s6k; cat. no. 2708; Cell Signaling Technology, Inc.), rabbit anti-p-p70s6k (cat. no. ab1314362; Abcam) and rabbit anti-GAPDH (cat. no. CW0101; Beijing ComWin Biotech Co., Ltd., Beijing, China) antibodies. All primary antibodies were diluted to 1:500. Following washing with $0.1 \%$ TBST, membranes were treated with a horseradish peroxidase-conjugated goat anti-rabbit immunoglobulin G secondary antibody (cat. no. CW0101, 1:2,000; Beijing ComWin Biotech Co., Ltd.) for 
$2 \mathrm{~h}$ at $37^{\circ} \mathrm{C}$. The signals were detected with enhanced chemiluminescence kit (EMD Millipore, Billerica, MA, USA). The relative densities of LC3, PI3K, p-PI3K, Rheb, Akt, p-Akt, p70s6k and p-p70s6k were determined by normalization to the density value of GAPDH in the same blot. Relative band intensity was analyzed using ImageJ 1.8.0 software (National Institutes of Health, Bethesda, MD, USA).

Reverse transcription-quantitative polymerase chain reaction $(R T-q P C R)$. Total RNA was extracted from cells using TRIzol reagent (Invitrogen; Thermo Fisher Scientific, Inc.), according to the manufacturer's protocol. miRNA was reverse transcribed into cDNA $\left(25^{\circ} \mathrm{C}\right.$ for $10 \mathrm{~min}, 42^{\circ} \mathrm{C}$ for $50 \mathrm{~min}$, followed by $70^{\circ} \mathrm{C}$ for $15 \mathrm{~min}$ ) using an miRNA cDNA synthesis kit (Beijing Aidiab Biotechnologies Co., Ltd., Beijing, China) and qPCR was performed with a SYBR Green miRNA qPCR assay kit (Beijing ComWin Biotech Co., Ltd., Beijing, China). mRNA was reverse transcribed $\left(25^{\circ} \mathrm{C}\right.$ for $10 \mathrm{~min}, 42^{\circ} \mathrm{C}$ for $50 \mathrm{~min}$, followed by $70^{\circ} \mathrm{C}$ for $15 \mathrm{~min}$ ) using a PrimeScript ${ }^{\mathrm{TM}}$ RT reagent kit (Beijing Aidiab Biotechnologies Co., Ltd.,) and qPCR was performed with SYBR ${ }^{\circledR}$ Premix Ex Taq ${ }^{\text {TM }}$ (Takara Bio, Inc., Otsu, Japan). The housekeeping gene GAPDH was used as an internal control; the internal control employed for the normalization of miRNA expression levels was U6 (Sangon Biotech Co., Ltd. Shanghai, China). PI3K, Rheb and GAPDH primers were purchased from Sangon Biotech Co., Ltd. The mature miR-155 primer (qRT-PCR Primer Set, cat. no. 132, Sanger Registry ID Human has-miR-155, Sanger Accession no. Human MIMAT0000646, mature sequence UUAAUGCUA AUCGUGAUAGGGGU) was purchased from Takara Bio, Inc. The sequences of each specific primer were as follows: PI3K forward, 5'-GACTTTGCGACAAGA CTGCC-3' and reverse, 5'-AATCTGAAGCAGCGCCTG AA-3'; Rheb forward, 5'-AGCTTTGGCAGAATCTTGGA-3' and reverse, 5'-CACATCACCGAGCATGAAGA-3'; GAPDH forward, 5'-AGAAGGCTGGGGCTCATTTG-3' and reverse, 5'-AGAAGGCTGGGGCTCATTTG-3; U6 forward, 5'-CTC GCTTCGGCAGCACA-3' and reverse, 5'-AACGCTTCACGA ATTTGCGT-3'. A total of three independent experiments were performed under the same experimental conditions. All reactions ran at $95^{\circ} \mathrm{C}$ for $2 \mathrm{~min}$, followed by 40 cycles at $94^{\circ} \mathrm{C}$ for $15 \mathrm{sec}$ and $60^{\circ} \mathrm{C}$ for $1 \mathrm{~min}$ and extension $75^{\circ} \mathrm{C}$ for $30 \mathrm{sec}$. The relative mRNA and miRNA expression levels were quantified by calculating the $\Delta \mathrm{Cq}$ value: $\mathrm{Cq}$ value (target gene)-Cq value (housekeeping gene). The relative expression levels were calculated using the $2^{-\Delta \Delta C q}$ method (17).

Bioinformatics analysis to predict target genes for miR-155. The present study used bioinformatics tools to predict target genes for miR-155, including miRanda (omictools.com/miranda-tool), TargetScan (www.targetscan.org), miRBase (www.mirbase.org) and PicTar (pictar.mdc-berlin.de). miR-155 was determined to be closely associated with the PI3K/Akt/mTOR signaling pathway. The gene PIK3CA, encoding the p110 catalytic protein subunit of PI3K, as well as Rheb, were identified as potential target genes of miR-155.

Plasmid construction and dual luciferase reporter assay. Human miR-155-5p overexpression plasmid, negative control plasmid, PIK3CA, and Rheb 3'-untranslated region (UTR) reporter plasmids were designed and purchased from Shanghai Genechem Co.,Ltd. (Shanghai,China). PIK3CA-wild type (wt), PIK3CA-mutant (mut), Rheb-wt and Rheb-mut reporters were successfully constructed using molecular cloning technology. For the construction of the PIK3CA- and Rheb-expressing plasmid, the 3'-UTRs of PIK3CA and Rheb, which contained the putative miR-155 binding sites, were amplified using PCR (conducted by Shanghai Genechem Co., Ltd.) and the product inserted into pGL3 luciferase plasmids at the NotI and XhoI restriction sites. The reporter vector was termed wt. A point mutation (mut) was incorporated into the binding sites of the 3'-UTR in the PIK3CA and Rheb genes to generate a mutant reporter vector. 293T cells grown in 24-well plates were co-transfected using Lipofectamine 2000 with $10 \mathrm{nM}$ negative controls or miR-155 mimics, and 500 ng dual-luciferase reporter plasmid per well, which was either PIK3CA-wt, PIK3CA-mut, Rheb-wt, Rheb-mut or empty vector. Renilla relative luciferase activity was measured $48 \mathrm{~h}$ post-transfection using a dual-luciferase reporter assay system according to the manufacturer's protocol (Promega Corporation, Madison, WI, USA). Each transfection was repeated three times.

Statistical analysis. Statistical analyses were performed with SPSS software version 16.0 (SPSS, Inc., Chicago, IL, USA). Differences between groups were assessed using one-way analysis of variance followed by Fisher's Least Significant Difference for multiple comparisons. The results were expressed as the mean \pm standard deviation of three independent experiments. $\mathrm{P}<0.05$ was considered to indicate a statistically significant difference.

\section{Results}

Overexpression of miR-155 induces autophagy. To explore the role of miR-155 in autophagy, the present study used TEM to evaluate autophagosome and autolysosome accumulation. The TEM results demonstrated that the average number of autophagic vacuoles and autolysosomes in the control group was 2-3. It was observed that inhibition of the expression of miR-155 with miR-155 inhibitors resulted in the suppressed formation of autophagic vacuoles and autolysosomes, compared with the control mimic (NC) group. The average number of autophagic vacuoles and autolysosomes was $0-1$ in the miR-155 inhibitor group. In the miR-155 mimic groups, cells displayed a higher number of autolysosomes and autophagic vesicles than the NC group, and the average number of autolysosomes and autophagic vacuoles was 5-7 (Fig. 1A). Following this, laser confocal microscopy was performed to detect LC3 puncta accumulation in ox-LDL-stimulated HUVECs. LC3 puncta appear in the cytoplasm and reflect the recruitment of $\mathrm{LC} 3$ proteins to autophagosomes (18). The fluorescence intensity of LC3 was reduced in miR-155 inhibitor-transfected cells, compared with NC cells. Conversely, transfection of HUVECs with miR-155 mimics induced an increase in the fluorescence intensity, compared with NC cells (Fig. 1B). To further confirm the promotion of autophagy by miR-155, the conversion of LC3-I to LC3-II (via the ratio of LCII to LC1) was examined through western blotting (Fig. 2A). It was observed that LC3-II expression levels were higher in the miR-155-mimic group compared with the control group, and that these autophagic markers were 
A
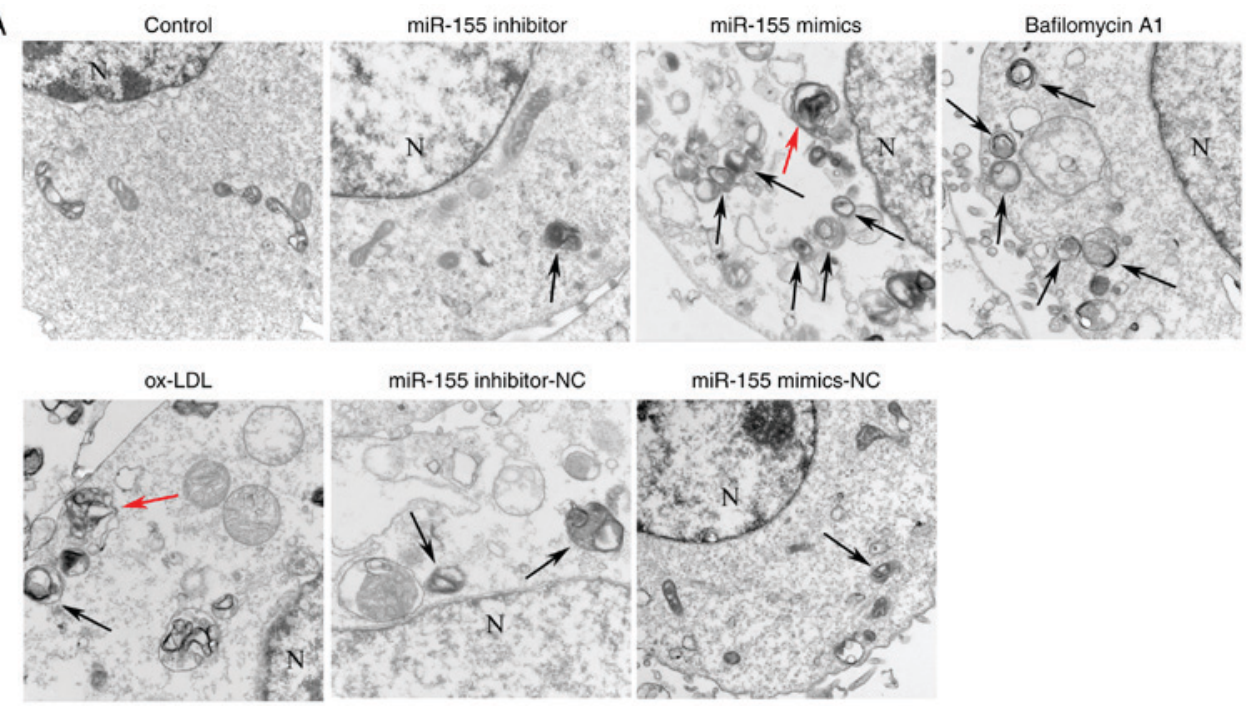

miR-155 inhibitor-NC

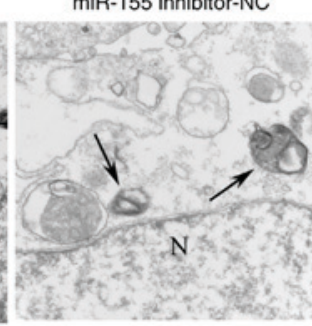

miR-155 mimics-NC

B
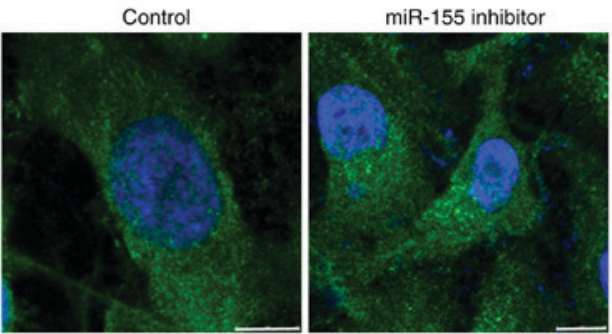

miR-155 mimics

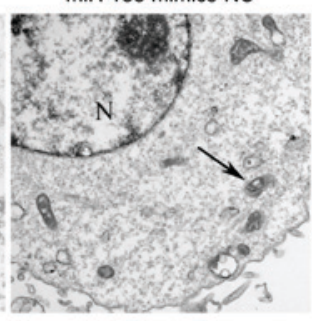

ox-LDL

miR-155 inhibitor-NC
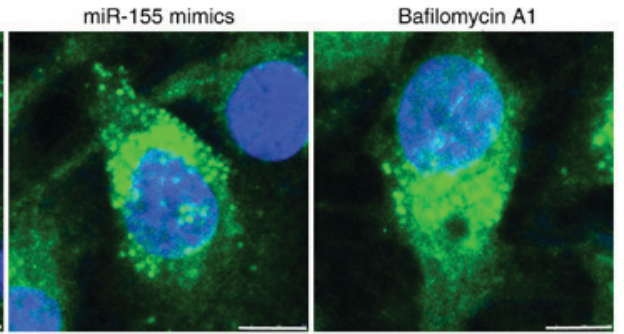

miR-155 mimics-NC
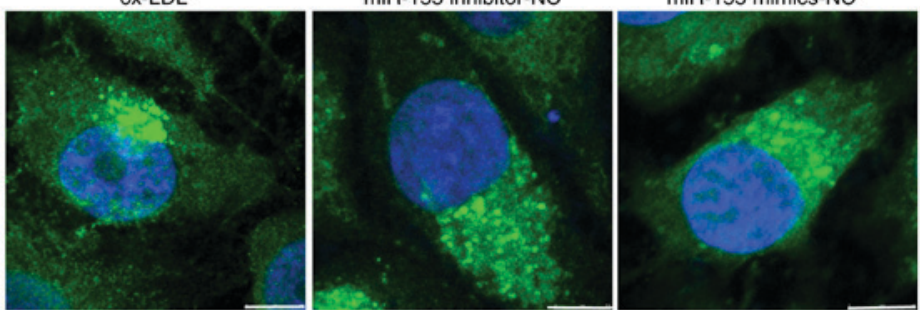

Figure 1. Overexpression of miR-155 induces autophagy. (A) Distribution of autophagosomes (black arrow) and autolysosomes (red arrow) in HUVECs were visualized using a transmission electron microscope in each group. Magnification, x40,000. (B) Confocal microscopy images of HUVECs. Endogenous light chain 3 protein expression was labeled with green fluorescence. Nuclei were labeled with DAPI (blue). Scale bar, $10 \mu$ m. HUVECs, human umbilical vein endothelial cells; N, nucleus; miR-155, microRNA-155; ox-LDL, oxidized low-density lipoprotein; NC, negative control.

inhibited in HUVECs transfected with miR-155-inhibitors, compared with the control group (Fig. 2B), which was in accordance with results from electron microscopy and confocal microscopy. Lysomotropic bafilomycin A1 prevents lysosome and autophagosome fusion, and is often used for measurement of autophagic flux (19). When cells were treated with bafilomycin A1, autophagic activity was significantly increased compared with the ox-LDL group, and the average number of autolysosomes and autophagic vacuoles was 5-6 (Figs. 1 and 2). Taken together, these results suggested that miR-155 efficiently promoted autophagy in vascular endothelial cells.

Overexpression of miR-155 promotes autophagy by suppressing the activation of the PI3K/Akt/mTOR signaling pathway in HUVECs. The efficiency of miR-155 transfection in HUVECs was examined. RT-qPCR data revealed that miR-155 expression was significantly increased in the miR-155 mimic group, compared with the corresponding negative control group. Furthermore, miR-155 levels were significantly decreased compared with the corresponding negative control group following miR-155 inhibitor transfection (Fig. 3A). The results were repeatedly verified as stable following the exclusion of interference from other factors under the same conditions, such as the transfection reagent and transfection conditions. Our previous study achieved similar results (16). To investigate the role of the PI3K/Akt/mTOR signaling pathway and miR-155 in HUVECs, RT-qPCR and western blotting was performed to detect the mRNA and protein expression levels of key members of the PI3K/Akt/mTOR signaling pathway. The results demonstrated that overexpression of miR-155 inhibited Akt (Fig. 3B) and p70s6k (Fig. 3C) mRNA expression, compared with the controls. Furthermore, Akt and p70s6k mRNA expression significantly increased in the miR-155 inhibitor group. In addition, western blot analysis was performed to determine the relative protein expression of total/p-Akt and total/p-p70s6k (Fig. 3D). Densitometry confirmed that similar, significant effects were observed in the levels of Akt (Fig. 3E) and p70s6k (Fig. 3F) activity in 

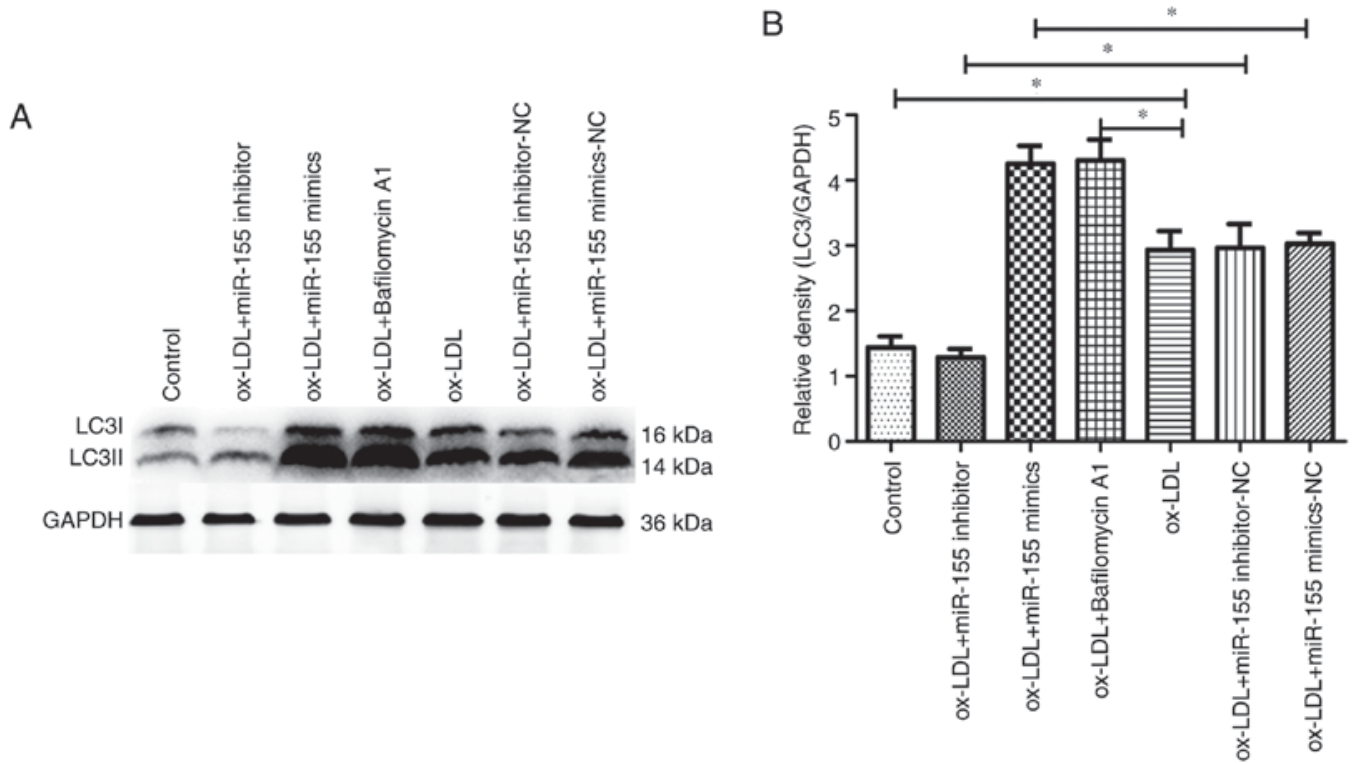

Figure 2. Effect of miR-155 on autophagy marker, LC3. (A) Representative images of the western blot analysis to determine the expression of LC3 in ox-LDL-treated human umbilical vein endothelial cells. GAPDH was used as an internal control. (B) Quantification of the LC3-II/LC3-I ratio. Data are expressed as the mean \pm standard deviation $(n=3)$. "P<0.05. miR-155, microRNA-155; ox-LDL, oxidized low-density lipoprotein; NC, negative control; LC3, microtubule-associated protein light chain 3.
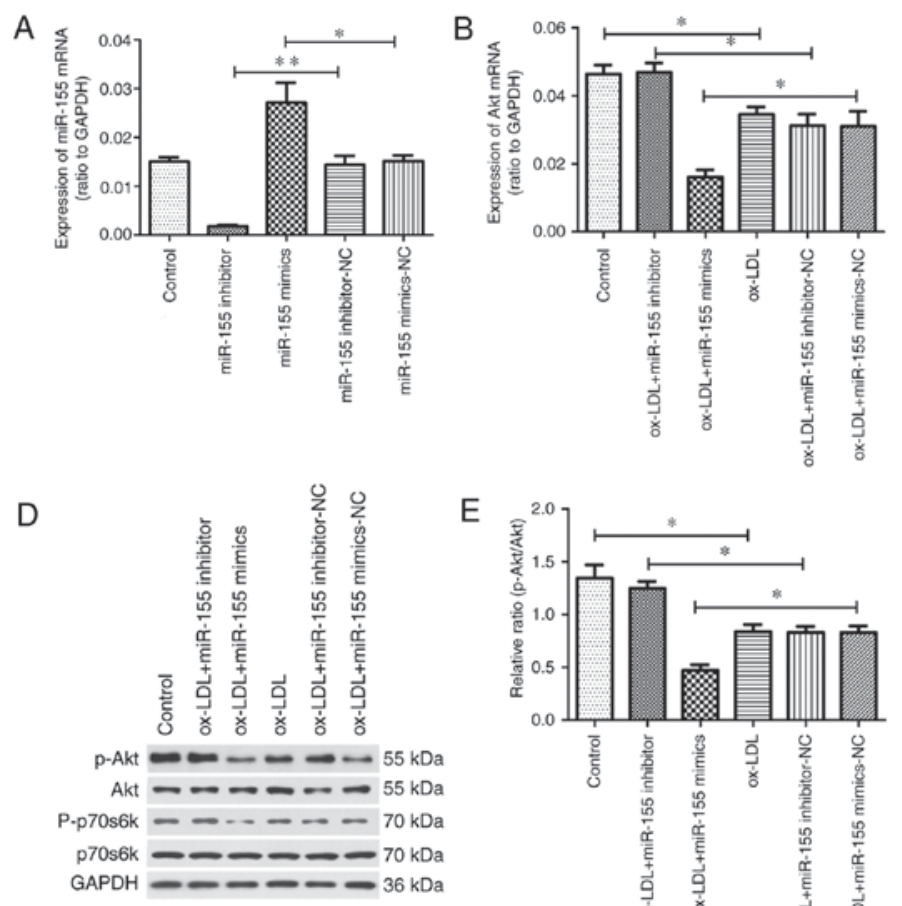

E

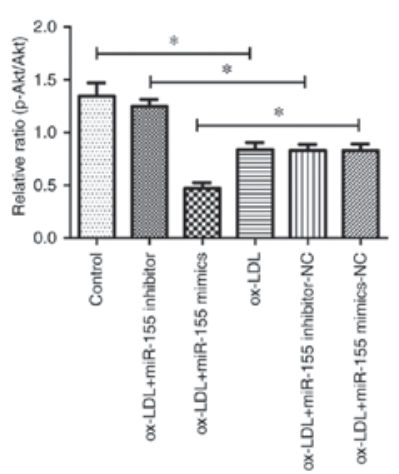

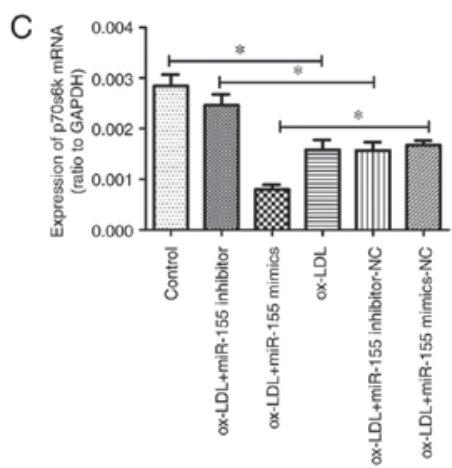

$\mathrm{F}$

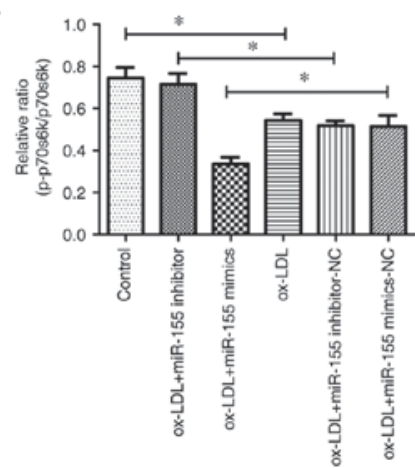

Figure 3. miR-155 suppresses the activation of the PI3K/Akt/mTOR signaling pathway. RT-qPCR was performed to analyze (A) miR-155 (U6 as a normalization internal control), (B) Akt and (C) p70s6k expression. (D) Representative image of western blot analysis to determine total Akt, p-Akt, p70s6k, p-p70s6k and GAPDH protein expression in ox-LDL-treated human umbilical vein endothelial cells transfected with miR-155 mimics, inhibitors or NCs. Quantification of western blotting results as a relative ratio of (E) p-Akt/Akt and (F) p-p70s6k/p70s6k expression. Data are expressed as the mean \pm standard deviation ( $\mathrm{n}=3$ ). ${ }^{*} \mathrm{P}<0.05 ;{ }^{* * *} \mathrm{P}<0.01$. miR-155, microRNA-155; ox-LDL, oxidized low-density lipoprotein; NC, negative control; p, phosphorylated; Akt, RAC- $\alpha$ serine/threonine-protein kinase; p70s6k, $70 \mathrm{kDa}$ ribosomal protein $\mathrm{S} 6$ kinase 1.

response to miR-155 mimics and inhibitors. Therefore, the data verified that miR-155 promoted autophagy by negatively regulating the expression PI3K/Akt/mTOR signaling pathway proteins in HUVECs.
miR-155 promotes autophagy by direct interaction with the 3'-UTRs of PIK3CA and Rheb. An association between miR-155 and the PI3K/Akt/mTOR signaling pathway was demonstrated in the present study; however, the present 
A

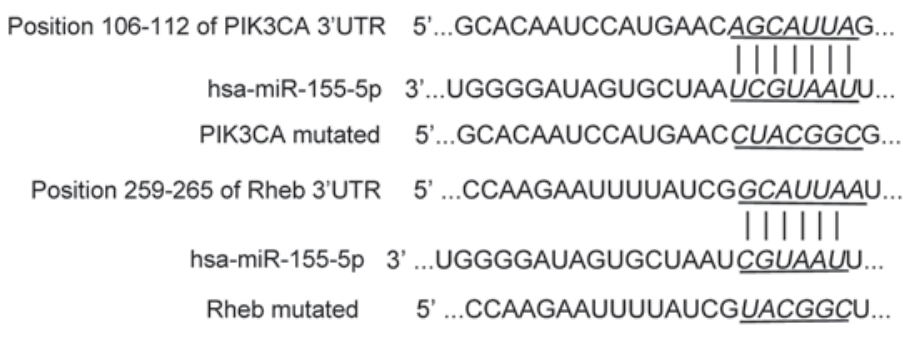

B

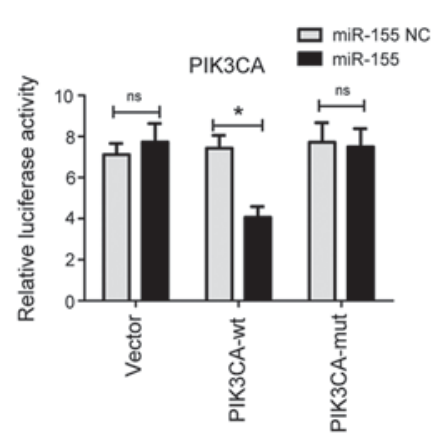

C

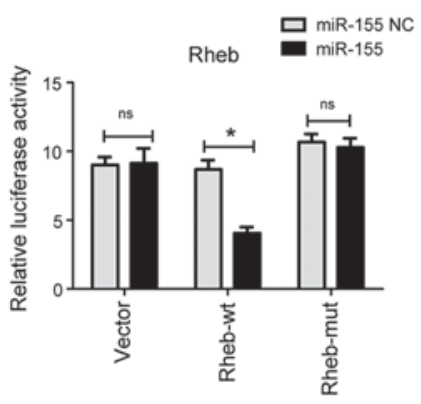

Figure 4. miR-155 targets PIK3CA and Rheb. (A) The predicted binding site of miR-155 in the 3'-UTR of PIK3CA and Rheb. 293T cells were transfected with a reporter vector containing the wt or mut (B) PIK3CA and (C) Rheb 3'-UTR, along with NC or miR-155 mimics. Firefly luciferase activity was normalized to Renilla luciferase. The results are presented as the mean \pm standard deviation, according to three independent experiments, $\mathrm{n}=3$ * $\mathrm{P}<0.05$. miR-155, microRNA-155; NC, negative control; UTR, untranslated region; wt, wild-type; mut, mutant; Rheb, Ras homolog enriched in brain; PIK3CA, phosphatidylinositol-3 kinase subunit $\alpha$.

study aimed to elucidate the mechanism underlying this. Bioinformatics analyses were performed to predict potential target genes for miR-155. PIK3CA and Rheb were identified due to the existence of the evolutionarily conserved miR-155 binding site in the 3'-UTR of these genes (Fig. 4A). In order to confirm these genes as targets of miR-155, a luciferase reporter plasmid was constructed that contained the human PIK3CA and Rheb mRNA 3'-UTR binding sites, downstream of the firefly luciferase reporter gene (the reporter vector was named wild-type). A point mutation was also incorporated into the PIK3CA and Rheb gene 3'-UTR binding sites to generate a mutant reporter vector.

miR-155 mimic or NC was transfected with $500 \mathrm{ng}$ wild-type or mutated reporter plasmids. Co-expression of PIK3CA or Rheb 3'-UTR constructs with miR-155 mimics was established in 293 cells. Luciferase activity was determined $24 \mathrm{~h}$ post-transfection. The luciferase reporter assay indicated that miR-155 significantly reduced the relative luciferase activity of the reporter vector containing the wild-type 3'-UTR of PIK3CA (Fig. 4B) or Rheb (Fig. 4C), compared with the negative control. Conversely, miR-155 did not significantly inhibit luciferase activity in cells expressing mutant PIK3CA and Rheb 3'-UTR, or empty vector. These results suggested that miR-155 directly bound to the 3'-UTR of PIK3CA and Rheb, and inhibited luciferase activity. Therefore, PIK3CA and Rheb were verified as target genes of miR-155.

miR-155 expression was subsequently detected in cells stimulated with ox-LDL. Ox-LDL treatment alone significantly increased miR-155 expression compared with the control. In addition, treatment with ox-LDL and miR-155 mimics resulted in significantly higher miR-155 expression, compared with the ox-LDL and miR-155 mimic NC group (Fig. 5A). In addition, the levels of PI3K (Fig. 5B) and Rheb (Fig. 5C) mRNA were significantly downregulated in HUVECs transfected with miR-155 mimics compared with the corresponding NC group. However, in cells stimulated with ox-LDL, miR-155 inhibitor transfection increased PI3K and Rheb mRNA expression to levels comparable with the control group. Furthermore, western blot analysis was performed to detect the protein expression of Rheb, PI3K and p-PI3K (Fig. 5D). Transfection of miR-155 mimics suppressed the protein levels of p-PI3K (Fig. 5E) and Rheb (Fig. 5F) compared with the control group; however, miR-155 inhibitors did not result in a significant reduction in protein levels. In addition, total PI3K expression remained unchanged. The different effects observed at the mRNA and proteins were thought to be due to two potential reasons. It may be a dynamic effect; one mRNA converts to protein more efficiently within a certain time, so protein downregulation is not significant. On the other hand, this may be due to a negative feedback loop in the cell. In this process, following miRNA-mediated protein downregulation, the cell may increase mRNA production due to the cell requirements, or other regulatory processes (20-25). These results verified that miR-155 suppressed the expression of PI3K and Rheb in HUVECs. Therefore, it is possible that miR-155 regulated autophagy by targeting PI3K and Rheb in atherosclerosis.

\section{Discussion}

The present study explored the underlying mechanisms of miR-155-regulated autophagy in vascular endothelial cells, using an in vitro model of HUVECs stimulated with ox-LDL. The primary findings of the current study were that: i) Overexpression of miR-155 upregulated autophagic activity 

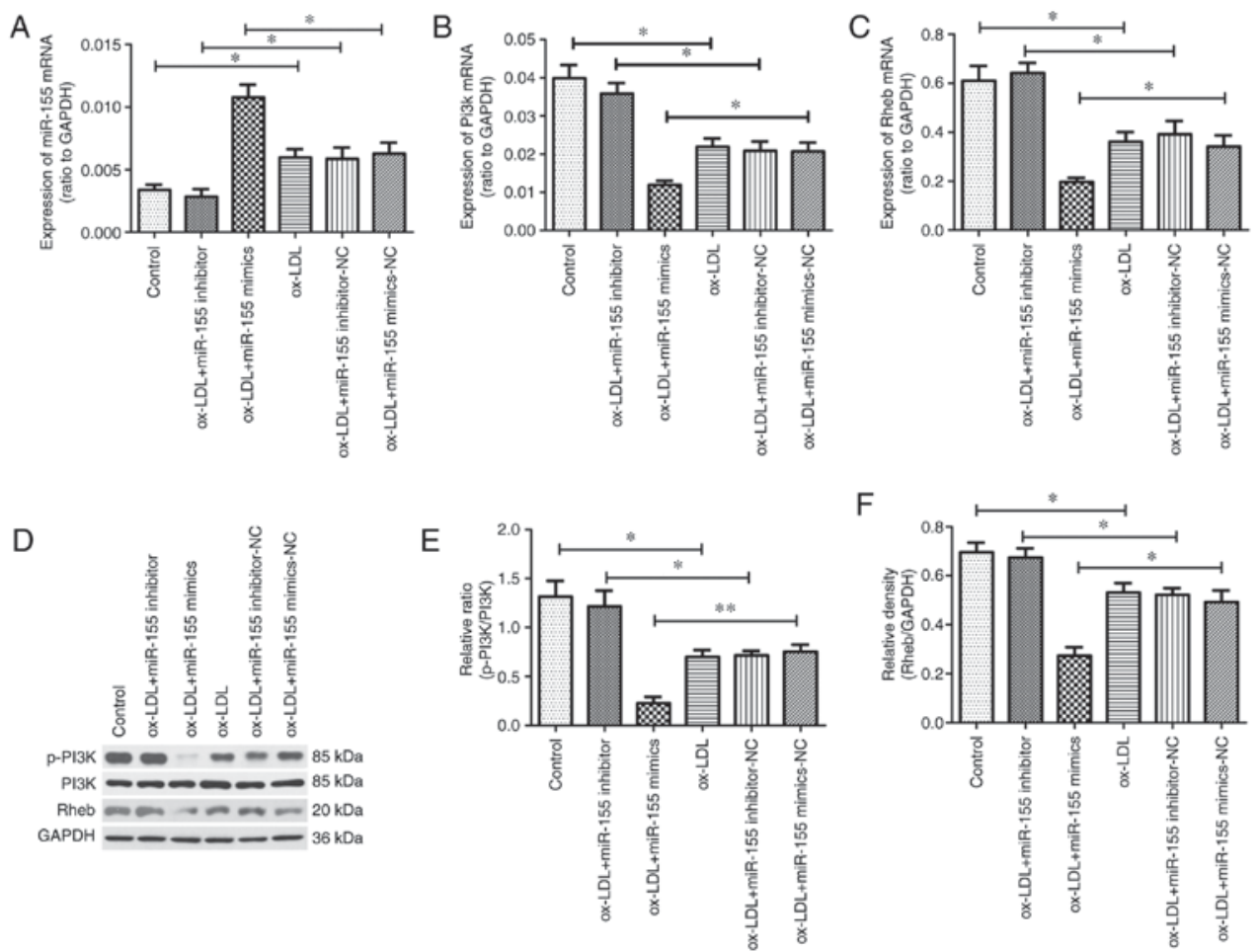

Figure 5. miR-155 regulates PI3K and Rheb expression. Human umbilical vein endothelial cells were treated with ox-LDL and transfected with miR-155 mimics, miR-155 inhibitor or NCs. Reverse transcription-quantitative polymerase chain reaction analysis of (A) miR-155, (B) PI3K and (C) Rheb mRNA expression. (D) Representative image of western blotting results. Quantification of western blotting results as relative ratio of (E) p-PI3K/PI3K and relative density of (F) Rheb. The density of p-PI3K and Rheb was normalized to GAPDH density in the same blot. Results are presented as the mean \pm standard deviation of three independent experiments. ${ }^{*} \mathrm{P}<0.05,{ }^{* *} \mathrm{P}<0.01$. miR-155, microRNA-155; ox-LDL, oxidized low-density lipoprotein; NC, negative control; $\mathrm{p}$, phosphorylated; PI3K, phosphatidylinositol-3 kinase; Rheb, Ras homolog enriched in brain.

and inhibition of miR-155 expression reduced autophagic activity; ii) overexpression of miR-155 promoted autophagy by suppressing the activation of the PI3K/Akt/mTOR signaling pathway; and iii) dual luciferase reporter assays confirmed PIK3CA and Rheb as target genes of miR-155. Therefore, it was established that miR-155-mediated inhibition of PI3K/Akt/mTOR signaling regulated autophagy, and that this occurred through targeting of PI3K and Rheb. This mechanism may be involved in the development of atherosclerosis.

miR-155 is one of the most extensively studied miRNAs, and has been demonstrated to be involved in regulating the cellular autophagy process. Chen et al (26) reported that an increase in miR-155 expression levels upregulates anticancer drug-induced autophagy in osteosarcoma cells. In addition, Liu et al (27) suggested that miR-155 alleviates septic lung injury by inducing autophagy via inhibition of TGF- $\beta$ activated kinase 1 (MAP3K7) binding protein 2. The results of the present study demonstrated that overexpression of miR-155 significantly increased autophagic activity in ox-LDL-induced HUVECs; however, suppression of miR-155 expression inhibited autophagic activity. These results implied that miR-155 may have an important role in the regulation of vascular endothelial cell autophagy, which makes miR-155 a potential target in atherosclerosis therapy.

$\mathrm{PI} 3 \mathrm{~K} / \mathrm{Akt} / \mathrm{mTOR}$ signaling is regarded as a classic autophagic signaling pathway, which transmits signals from the cell membrane to the nucleus and activates multiple cellular events (28). Previously, the PI3K/Akt/mTOR signaling pathway has been reported to serve an important role in the regulation of autophagy in atherosclerosis $(9,10)$. PI3K activates the phosphorylation of Akt, followed by the activation of mTOR to finally trigger the inhibition of autophagy. P70s6k is a key downstream target of mTOR, and an essential target for the regulation of protein translation and additional metabolic processes in cell growth (29). Activation of mTOR complex 1 triggers a cascade of anabolic processes for cell growth, proliferation and autophagy, primarily mediated by p70s6k (30). To verify whether miR-155 regulates autophagy via the PI3K/Akt/mTOR pathway, the present study performed RT-qPCR and western blot analysis in ox-LDL-stimulated HUVECs to determine the expression of key proteins of the PI3K/Akt/mTOR signaling pathway, including Akt, p-Akt, p70s6k and p-p70s6k. It was demonstrated that Akt and p70s6k expression was repressed when miR-155 mimics were transfected into ox-LDL-treated HUVECs; however, inhibition of miR-155 increased the activation of Akt and p70s6k. The results of the present study therefore revealed that miR-155 downregulated Akt and p70s6k at both the mRNA and protein level in HUVECs. The PI3K/Akt/mTOR signaling pathway negatively regulates autophagy (31), as evidenced by the upregulation of autophagy induced by the inhibition of Akt and p70s6k in the preset study. This suggests that miR-155 promotes autophagy by suppressing the PI3K/Akt/mTOR pathway, and this may be involved in the regulation of atherosclerosis.

The present study focused on the PI3K/Akt/mTOR signaling pathway in atherosclerosis, and investigated whether miR-155 regulated autophagy in vascular endothelial cells 
by targeting key proteins of the PI3K/Akt/mTOR signaling pathway. Bioinformatics tools predicted that PIK3CA and downstream protein Rheb were potential target genes of miR-155. A dual luciferase reporter assay demonstrated that miR-155 downregulated PIK3CA and Rheb by directly targeting the 3 '-UTR region of these genes. The inhibitory effect of miR-155 on the expression of PI3K and Rheb protein and mRNA was also analyzed by western blot analysis and RT-qPCR assays, respectively. These results again revealed the role of miR-155 in the PI3K/Akt/mTOR pathway, and suggested that PI3K and Rheb are direct targets of miR-155 that may be involved in the pathogenesis of atherosclerosis. Huang et al (32) observed that miR-155 activates the PI3K-Akt signaling pathway by targeting PI3K regulatory subunit $\mathrm{p} 85 \alpha$ in diffuse large B-cell lymphoma. A study by Wan et al (33) suggested that hypoxia-induced expression of miR-155 occurs in human cervical cancer and nasopharyngeal cancer cells, which suppresses the expression of multiple target genes in the mTOR signaling pathway, including Rheb, RPTOR independent companion of MTOR complex 2 and ribosomal protein S6 kinase B2. In addition, knockdown of endogenous miR-155 expression inhibits hypoxia-induced autophagy (33). Wang et al (34) demonstrated that miR-155 promotes autophagy by targeting Rheb to eliminate intracellular mycobacteria. These studies provide evidence that miR-155 activates autophagy through the PI3K/Akt/mTOR signaling pathway. Further study is required to determine whether other autophagy-associated pathways also participate in this process.

In conclusion, the present study demonstrated that miR-155 downregulated the activity of the PI3K/Akt/mTOR signaling pathway via targeting PI3K and Rheb, to promote autophagy in ox-LDL-induced HUVECs. Therefore, miR-155 may be a potential therapeutic target in atherosclerosis. The present study identified a novel role for miR-155 in the regulation of endothelial cell autophagy during atherosclerosis, and provided insight into the future development of innovative atherosclerosis therapies that may function by targeting miR-155.

\section{Acknowledgements}

We sincerely thank the staff of Central Laboratory of the Affiliated Hospital of Qingdao University (Qingdao, China) and all members of our research group for general support.

\section{Funding}

The present study was supported by the National Natural Science Foundation of China (grant nos. 81571112 and 81641046), the Shandong Province Natural Science Foundation (grant no. 2015GSF118172) and the Municipal Scientific and Technological Project of Qingdao City (grant no. 15-9-2-86-nsh).

\section{Availability of data and materials}

The datasets used and/or analyzed during the current study are available from the corresponding author on reasonable request.

\section{Authors' contributions}

XP, AM and SYa designed the experiments; SYi, JM, HPe YD, SL, WL and XB performed experiments; SYi and AM analyzed the data; SYi and SYa wrote the paper.

\section{Ethics approval and consent to participate}

The present study was approved by the Ethics Committee of the Affiliated Hospital of Qingdao University.

\section{Patient consent for publication}

Not applicable.

\section{Competing interests}

The authors declare that they have no competing interests.

\section{References}

1. Zhong LX, Zhang Y, Wu ML, Liu YN, Zhang P, Chen XY, Kong QY, Liu J and Li H: Resveratrol and STAT inhibitor enhance autophagy in ovarian cancer cells. Cell Death Discov 2: 15071, 2016.

2. Tang F, Yang TL, Zhang Z, Li XG, Zhong QQ, Zhao TT and Gong L: MicroRNA-21 suppresses ox-LDL-induced human aortic endothelial cells injuries in atherosclerosis through enhancement of autophagic flux: Involvement in promotion of lysosomal function. Exp Cell Res 359: 374-383, 2017.

3. Ding Z, Liu S, Wang X, Dai Y, Khaidakov M, Romeo F and Mehta JL: LOX-1, oxidant stress, mtDNA damage, autophagy, and immune response in atherosclerosis. Can J Physiol Pharmacol 92: 524-530, 2014

4. Ding WX: Uncoupling AMPK from autophagy: A foe that hinders the beneficial effects of metformin treatment on metabolic syndrome-associated atherosclerosis? Focus on 'glucose and palmitate uncouple AMPK from autophagy in human aortic endothelial cells'. Am J Physiol Cell Physiol 308: C246-C248, 2015.

5. Shafique E, Choy WC, Liu Y, Feng J, Cordeiro B, Lyra A, Arafah M, Yassin-Kassab A, Zanetti AV, Clements RT, et al: Oxidative stress improves coronary endothelial function through activation of the pro-survival kinase AMPK. Aging (Albany NY) 5: 515-530, 2013.

6. Perrotta I and Aquila S: The role of oxidative stress and autophagy in atherosclerosis. Oxid Med Cell Longev 2015: $130315,2015$.

7. Mollace V, Gliozzi M, Musolino V, Carresi C, Muscoli S, Mollace R, Tavernese A, Gratteri S, Palma E, Morabito C, et al: Oxidized LDL attenuates protective autophagy and induces apoptotic cell death of endothelial cells: Role of oxidative stress and LOX-1 receptor expression. Int J Cardiol 184: 152-158, 2015.

8. Butler DE, Marlein C, Walker HF, Frame FM, Mann VM, Simms MS, Davies BR, Collins AT and Maitland NJ: Inhibition of the PI3K/AKT/mTOR pathway activates autophagy and compensatory Ras/Raf/MEK/ERK signalling in prostate cancer. Oncotarget 8: 56698-56713, 2017.

9. Jiang Y, Kou J, Han X, Li X, Zhong Z, Liu Z, Zheng Y, Tian Y and Yang L: ROS-dependent activation of autophagy through the $\mathrm{PI} 3 \mathrm{~K} / \mathrm{Akt} / \mathrm{mTOR}$ pathway is induced by hydroxysafflor yellow a-sonodynamic therapy in THP-1 macrophages. Oxid Med Cell Longev 2017: 8519169, 2017.

10. Zhai C, Cheng J, Mujahid H, Wang H, Kong J, Yin Y,Li J,Zhang Y, Ji X and Chen W: Selective inhibition of PI3K/Akt/mTOR signaling pathway regulates autophagy of macrophage and vulnerability of atherosclerotic plaque. PLoS One 9: e90563, 2014.

11. Gupta R, Arkatkar T, Keck J, Koundinya GK, Castillo K, Hobel S, Chambers JP, Yu JJ, Guentzel MN, Aigner A, et al: Antigen specific immune response in Chlamydia muridarum genital infection is dependent on murine microRNAs-155 and -182 . Oncotarget 7: 64726-64742, 2016. 
12. Zhang T, Tian F, Wang J, Jing J, Zhou SS and Chen YD Endothelial cell autophagy in atherosclerosis is regulated by miR-30-mediated translational control of ATG6. Cell Physiol Biochem 37: 1369-1378, 2015.

13. Wang B, Zhong Y, Huang D and Li J: Macrophage autophagy regulated by miR-384-5p-mediated control of Beclin-1 plays a role in the development of atherosclerosis. Am J Transl Res 8: 606-614, 2016

14. Ouimet M, Ediriweera H, Afonso MS, Ramkhelawon B, Singaravelu R, Liao X, Bandler RC, Rahman K, Fisher EA, Rayner KJ, et al: microRNA-33 regulates macrophage autophagy in atherosclerosis. Arterioscler Thromb Vasc Biol 37: 1058-1067, 2017.

15. Chistiakov DA, Orekhov AN and Bobryshev YV: Chemokines and relevant microRNAs in the atherogenic process. Mini Rev Med Chem 18: 597-608, 2018.

16. Zhang Z, Pan X, Yang S, Ma A, Wang K, Wang Y, Li T and Liu S: miR-155 promotes ox-LDL-induced autophagy in human umbilical vein endothelial cells. Mediators Inflamm 2017: 9174801, 2017.

17. Livak KJ and Schmittgen TD: Analysis of relative gene expression data using real-time quantitative PCR and the 2(-Delta Delta C(T)) method. Methods 25: 402-408, 2001.

18. Wang L, Chen M, Yang J and Zhang Z: LC3 fluorescent puncta in autophagosomes or in protein aggregates can be distinguished by FRAP analysis in living cells. Autophagy 9: 756-769, 2013.

19. Lee WK, Probst S, Santoyo-Sánchez MP, Al-Hamdani W, Diebels I, von Sivers JK, Kerek E, Prenner EJ and Thévenod F: Initial autophagic protection switches to disruption of autophagic flux by lysosomal instability during cadmium stress accrual in renal NRK-52E cells. Arch Toxicol 91: 3225-3245, 2017.

20. Zhao Q, Liu H, Yao C, Shuai J and Sun X: Effect of dynamic interaction between microRNA and transcription factor on gene expression. Biomed Res Int 2016: 2676282, 2016.

21. Olaru AV, Selaru FM, Mori Y, Vazquez C, David S, Paun B, Cheng Y, Jin Z, Yang J, Agarwal R, et al: Dynamic changes in the expression of MicroRNA-31 during inflammatory bowel disease-associated neoplastic transformation. Inflamm Bowel Dis 17: 221-231, 2011.

22. O'Donnell KA, Wentzel EA, Zeller KI, Dang CV and Mendell JT: c-Myc-regulated microRNAs modulate E2F1 expression. Nature 435: 839-843, 2005.

23. Yamakuchi M and Lowenstein CJ: MiR-34, SIRT1 and p53: The feedback loop. Cell Cycle 8: 712-715, 2009.
24. Liu H, Lin H, Zhang L, Sun Q, Yuan G, Zhang L, Chen S and Chen Z: miR-145 and miR-143 regulate odontoblast differentiation through targeting Klf4 and Osx genes in a feedback loop. J Biol Chem 288: 9261-9271, 2013

25. Wei X, Cheng X, Peng Y, Zheng R, Chai J and Jiang S: STAT5a promotes the transcription of mature mmu-miR-135a in 3T3-L1 cells by binding to both miR-135a-1 and miR-135a-2 promoter elements. Int J Biochem Cell Biol 77: 109-119, 2016.

26. Chen L, Jiang K, Jiang $\mathrm{H}$ and Wei P: miR-155 mediates drug resistance in osteosarcoma cells via inducing autophagy. Exp Ther Med 8: 527-532, 2014.

27. Liu F, Nie C, Zhao N, Wang Y, Liu Y, Li Y, Zeng Z, Ding C, Shao Q, Qing C, et al: MiR-155 alleviates septic lung injury by inducing autophagy via inhibition of transforming growth factor- $\beta$-activated binding protein 2 . Shock 48: 61-68, 2017.

28. Zeng S, Song H, Chen Y, Xie W and Zhang L: $\mathrm{B}_{7}-\mathrm{H}_{4}$-mediated immunoresistance is supressed by $\mathrm{PI}_{3} \mathrm{~K} / \mathrm{Akt} / \mathrm{mTOR}$ pathway inhibitors. Mol Biol 50: pp887-894, 2016.

29. Cheng KY and Hao M: Mammalian target of rapamycin (mTOR) regulates transforming growth factor- $\beta 1$ (TGF- $\beta 1$ )-induced epithelial-mesenchymal transition via decreased pyruvate kinase M2 (PKM2) expression in cervical cancer cells. Med Sci Monit 23: 2017-2028, 2017.

30. Nivon M, Richet E, Codogno P, Arrigo AP and Kretz-Remy C: Autophagy activation by NFkappaB is essential for cell survival after heat shock. Autophagy 5: 766-783, 2009.

31. Shintani T and Klionsky DJ: Autophagy in health and disease: A double-edged sword. Science 306: 990-995, 2004

32. Huang X, Shen Y, Liu M, Bi C, Jiang C, Iqbal J, McKeithan TW, Chan WC, Ding SJ and Fu K: Quantitative proteomics reveals that miR-155 regulates the PI3K-AKT pathway in diffuse large B-cell lymphoma. Am J Pathol 181: 26-33, 2012.

33. Wan G, Xie W, Liu Z, Xu W, Lao Y, Huang N, Cui K, Liao M, He J, Jiang Y, et al: Hypoxia-induced MIR155 is a potent autophagy inducer by targeting multiple players in the MTOR pathway. Autophagy 10: 70-79, 2014.

34. Wang J, Yang K, Zhou L, Minhaowu, Wu Y, Zhu M, Lai X, Chen T, Feng L, Li M, et al: MicroRNA-155 promotes autophagy to eliminate intracellular mycobacteria by targeting Rheb. PLoS Pathog 9: e1003697, 2013.

This work is licensed under a Creative Commons Attribution-NonCommercial-NoDerivatives 4.0 International (CC BY-NC-ND 4.0) License. 